\title{
A case of indirect cauda equina syndrome from metastatic prostate cancer
}

\author{
Shilo Lefresne, BSC, $M D(C) ;^{*}$ Alysa Fairchild, MD, FRCPC; ${ }^{*}$ Aalo Bistritz, MD, FRCPC; ${ }^{*}$ \\ Peter Venner, MD, FRCPC; ${ }^{\dagger}$ Don Yee, MD, FRCPC ${ }^{*}$
}

\begin{abstract}
We report the case of a patient with metastatic hormone refractory prostate cancer in whom "indirect" cauda equina syndrome developed concurrent with multilevel spinal cord compression (SCC). Three months after his first positive bone scan, a 65-yearold otherwise healthy man presented with severe back pain, bilateral lower extremity paresthesias, leg weakness and urinary retention. Magnetic resonance imaging (MRI) showed a dural-based mass causing SCC at the T9, T10 and T11 vertebrae, with a normal cauda equina. He received corticosteroids and palliative external beam radiotherapy, resulting in good pain control and gradual improvement in his neurological symptoms. He did well for 8 months, at which time his residual bilateral leg weakness abruptly worsened and he experienced numbness, paresthesias, urinary incontinence and constipation. Repeat MRI showed progression of epidural metastatic disease compressing the spinal cord or thecal sac at 7 thoracic vertebral levels. The cauda equina was also distorted and flattened without evidence of direct solid tumour impingement. We hypothesized that the etiology was increased intrathecal pressure due to disrupted cerebrospinal fluid flow resulting from multiple levels of upstream thecal sac compression. It is essential to image the entire spinal cord and cauda equina when patients with metastatic bone disease present with neurological symptoms to institute correct treatment and preserve function and mobility.
\end{abstract}

Can Urol Assoc J 2009;3(4):E31-E35

\section{Introduction}

Prostate cancer frequently metastasizes to bone, with this complication developing in $65 \%-75 \%$ of patients. ${ }^{1}$ As osteoblastic lesions are commonly found in the spine, this patient population is at high risk for malignant spinal cord compression (SCC) or cauda equina syndrome (CES). Up to $8 \%$ of prostate cancer patients will experience SCC at some point in the course of their disease; ${ }^{2}$ however, the incidence of CES in patients with prostate cancer is unknown as it is rarely reported separately from SCC. ${ }^{3,4}$

Neurological sequelae of malignant SCC or CES are usually due to direct pressure by a bony metastatic deposit, pathological fracture, an intramedullary lesion or a combination of these factors. ${ }^{5}$ Both oncological emergencies require early diagnosis and treatment to prevent the development of devastating and potentially irreversible neurological complications. We present a unique case of CES secondary to increased cerebrospinal fluid (CSF) pressure as a result of multilevel upstream malignant SCC in a patient with prostate cancer.

\section{Case report}

A previously healthy 61 -year-old man received a diagnosis of prostate carcinoma in 2002 after undergoing prostate biopsy for a prostate-specific antigen (PSA) level of $16 \mu \mathrm{g} / \mathrm{L}$. He underwent radical prostatectomy and bilateral pelvic lymph node sampling in January 2003 for a pT3bN0 adenocarcinoma; he had a Gleason score of $3+4=7$ with a tertiary component Gleason score of 5. Lymphvascular invasion, a positive left apical margin and bilateral extracapsular invasion were reported. His PSA level reached a nadir of $<0.02 \mu \mathrm{g} / \mathrm{L}$ by March 2003 (Fig. 1).

By December 2003, his PSA level had increased to $0.46 \mu \mathrm{g} / \mathrm{L}$. After a negative bone scan, he received $66 \mathrm{~Gy}$ in 33 daily fractions of salvage prostate bed radiotherapy (RT) in 2004 and experienced a PSA response. However, by June 2005, his PSA level had climbed to $2.0 \mu \mathrm{g} / \mathrm{L}$, and leutenizing hormone release hormone $(\mathrm{LHRH})$ agonist therapy was initiated in September 2005. Asymptomatic bone metastases developed in May 2006, with bone scan demonstrating multiple areas of uptake in the axial skeleton, and the patient was determined to be hormone refractory.

In August 2006, severe back pain developed and the patient was referred to radiation oncology. At that time, he also described bilateral lower extremity (LE) paresthesias, urinary retention and leg weakness requiring a wheelchair for mobility. Physical examination revealed abnormal sensation in all LE dermatomes, with a sensory level at the T10 level. Strength in all LE muscle groups using the Medical Research Council Rating of Muscle Strength ${ }^{6}$ was 3/5, with the left LE slightly weaker than the right. Knee 
and achilles deep tendon reflexes (DTRs) were brisk bilaterally $(3+/ 4)$ and plantar reflexes were equivocal. His PSA was $650 \mu \mathrm{g} / \mathrm{L}$.

Magnetic resonance imaging (MRI) of the entire spine revealed widespread bone metastases and a dural-based mass extending from the level of the T9/T10 disc space to the midT11 vertebral body measuring $5.4 \mathrm{~cm}$ craniocaudally (Fig. 2). The spinal cord in this region was anteriorly displaced and compressed to an anteroposterior (AP) diameter of $5 \mathrm{~mm}$. The conus and cauda equina were normal (Fig. 3). Clinical findings correlated well with radiological abnormalities.

Dexamethasone (4 mg 4 times daily) and emergent radiotherapy (20 Gy in 5 daily fractions) to the thoracic spine were initiated. Over the next 8 months the patient experienced good pain control and gradual improvement in leg strength (right more than left). He could ambulate with a walker, and he had return of bladder control and almost complete normalization of LE sensation. He remained hyperreflexic at the knees and ankles. He subsequently received systemic docetaxel-based therapy as a participant of a clinical trial from October 2006 through March 2007. His participation in the study was discontinued owing to clinical and biochemical progression.

In March 2007, acute-onset bilateral leg weakness (right worse than left), numbness and paresthesias below the knees bilaterally and urinary incontinence requiring catheterization developed. Back pain was not a predominant symptom. His
PSA level had risen to $1250 \mu \mathrm{g} / \mathrm{L}$. The patient was urgently reassessed by radiation oncology. Pain with palpation was present within a hyperpigmented region over the thoracic spine in the area of previous RT. Right leg muscle strength was $1 / 5$ proximally and $2 / 5$ distally. Left leg muscle strength was $2 / 5$ proximally and $3 / 5$ distally. Sensation in the LE was decreased from the feet to the knees bilaterally. Patellar and achilles DTRs remained hyperreflexic and Babinski sign was present bilaterally.

Repeat spinal MRI demonstrated diffuse marrow infiltration consistent with metastatic disease. Multiple epidural masses causing SCC were visible at the T1, T2, T8, T9 and T10 vertebral levels. We observed impending SCC as a consequence of the reduced diameter of the spinal canal at the T11 and T12 vertebral levels (Fig. 4). We also detected neural foraminal tumour extension at the T3/T4, T12/L1 and L4/L5 vertebral levels. The cauda equina was distorted and flattened without evidence of direct tumour compression. Axial images suggested that CSF was compressing the cauda equina in the AP plane (Fig. 4).

The patient received dexamethasone (4 mg 4 times daily) and palliative RT to the $\mathrm{C} 7-\mathrm{T} 3$ and $\mathrm{T} 7-\mathrm{L} 1$ vertebrae as an inpatient. He achieved good pain control, but despite intensive rehabilitation and supportive care, his neurological status did not improve. We discharged him home with 24-hour nursing care, but his condition deteriorated rapidly and he died in April 2007.

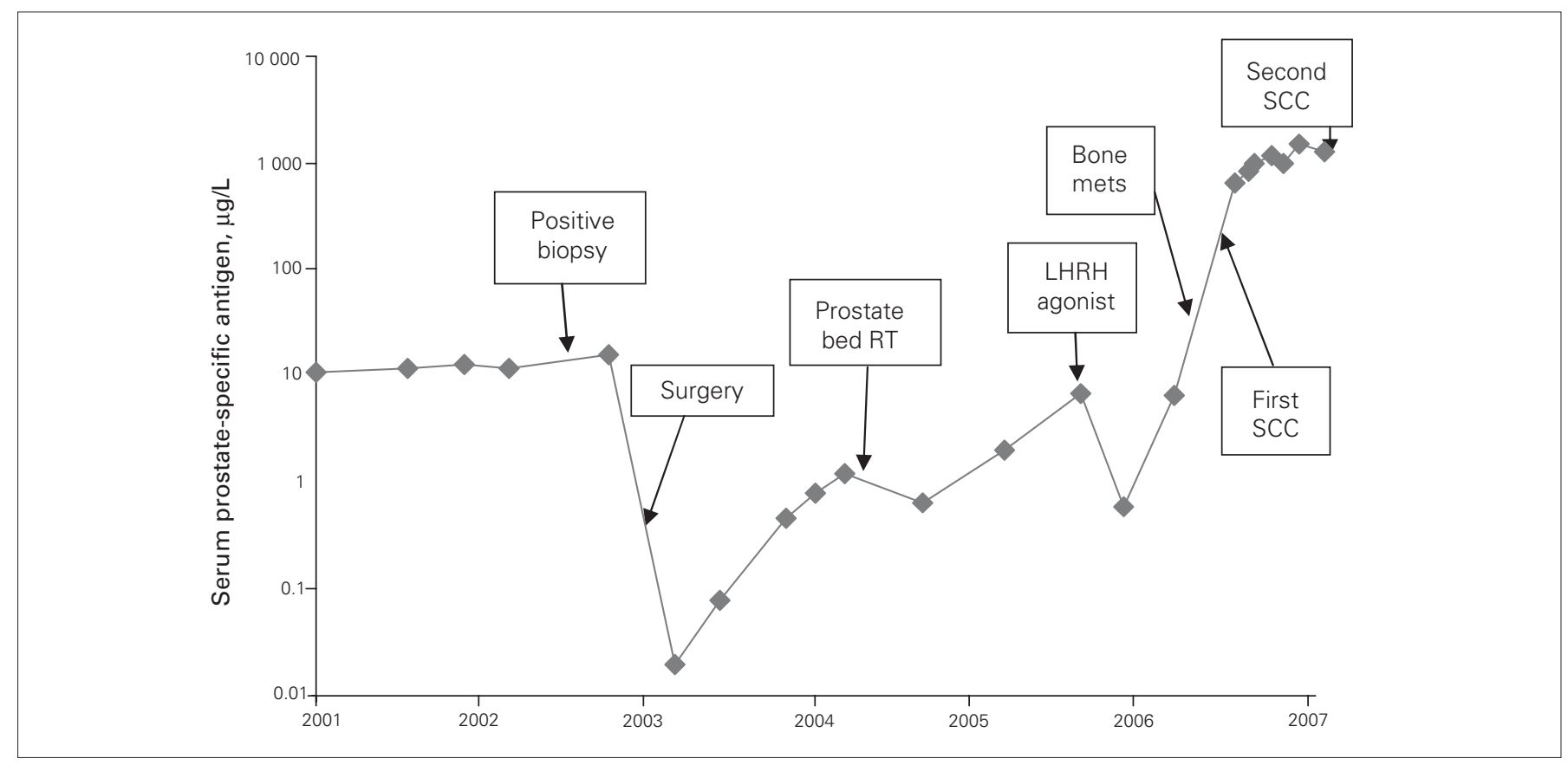

Fig. 1. Prostate-specific antigen (PSA) levels over time (log scale). LHRH = leutenizing hormone release hormone; mets = metastases; $R T=$ radiotherapy; SCC $=$ spinal cord compression. 


\section{Discussion}

We present the case of a prostate cancer patient with multilevel malignant SCC and an indirect CES, which likely contributed to his symptom profile. The patient's initial presentation with back pain and neurological symptoms was typical of SCC, and his clinical symptoms and physical exam correlated well with MRI findings. The most common SCC
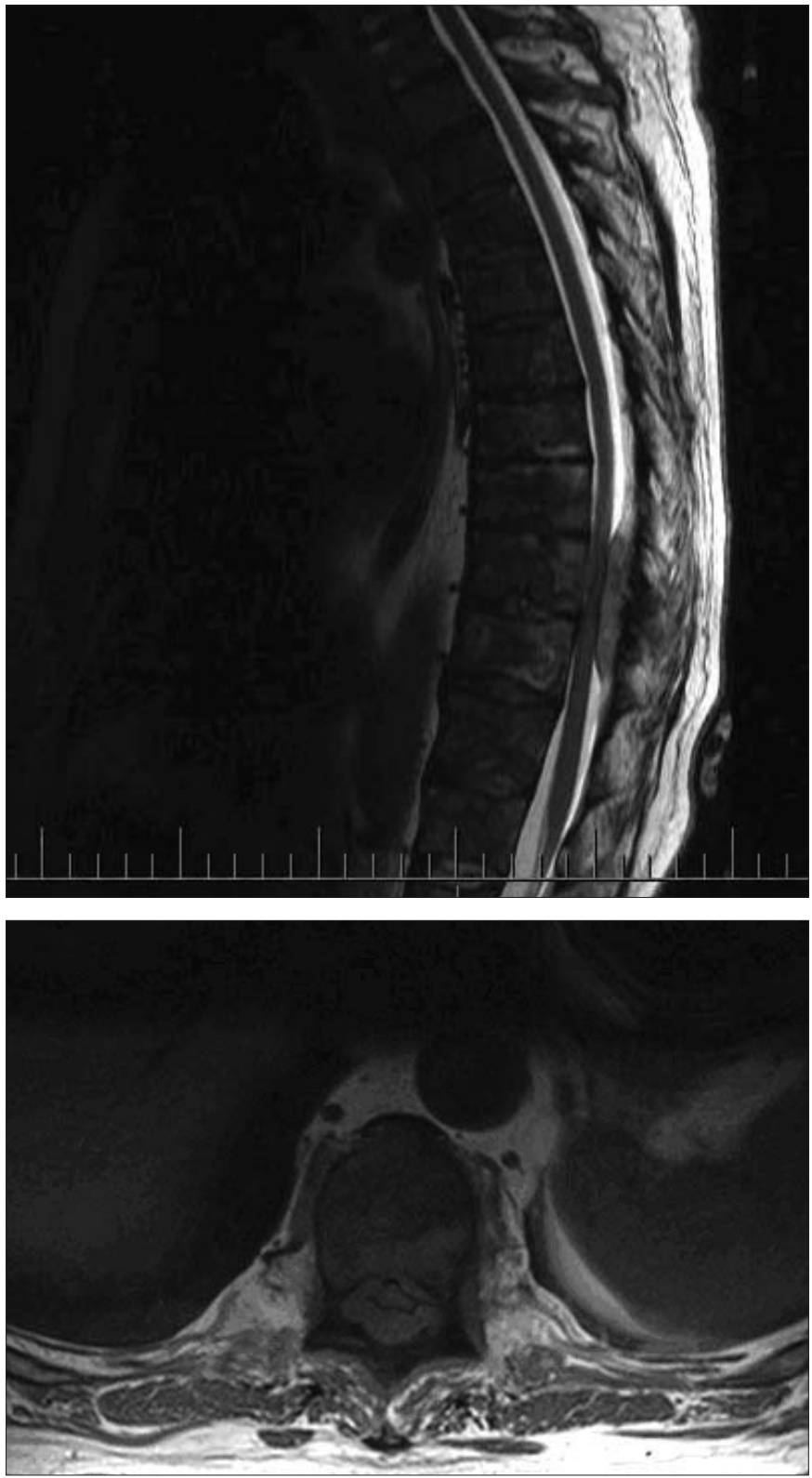

Fig. 2. Sagittal $T_{2}$-weighted magnetic resonance image (MRI) of the thoracic spine demonstrating spinal cord compression at the levels of the T10 and T11 vertebral bodies (top). Axial $T_{1}$-weighted MRI of the thoracic spine demonstrating spinal cord compression (bottom) (August 2006). symptoms are back pain, weakness, sensory changes, sphincter dysfunction and radiculopathy, ${ }^{5,7}$ and our patient had all but radiculopathy.

Our patient's recurrent SCC is not unique: up to $10 \%$ of patients experience recurrence. ${ }^{5}$ However, his second presentation had atypical clinical and radiological features, most notably the cauda equina compression in the absence of direct tumour involvement. Cauda equina syndrome secondary to metastatic prostate cancer is rare, ${ }^{3,4}$ but cauda equina compression in the absence of a visible tumour, to our knowledge, has not been previously reported.
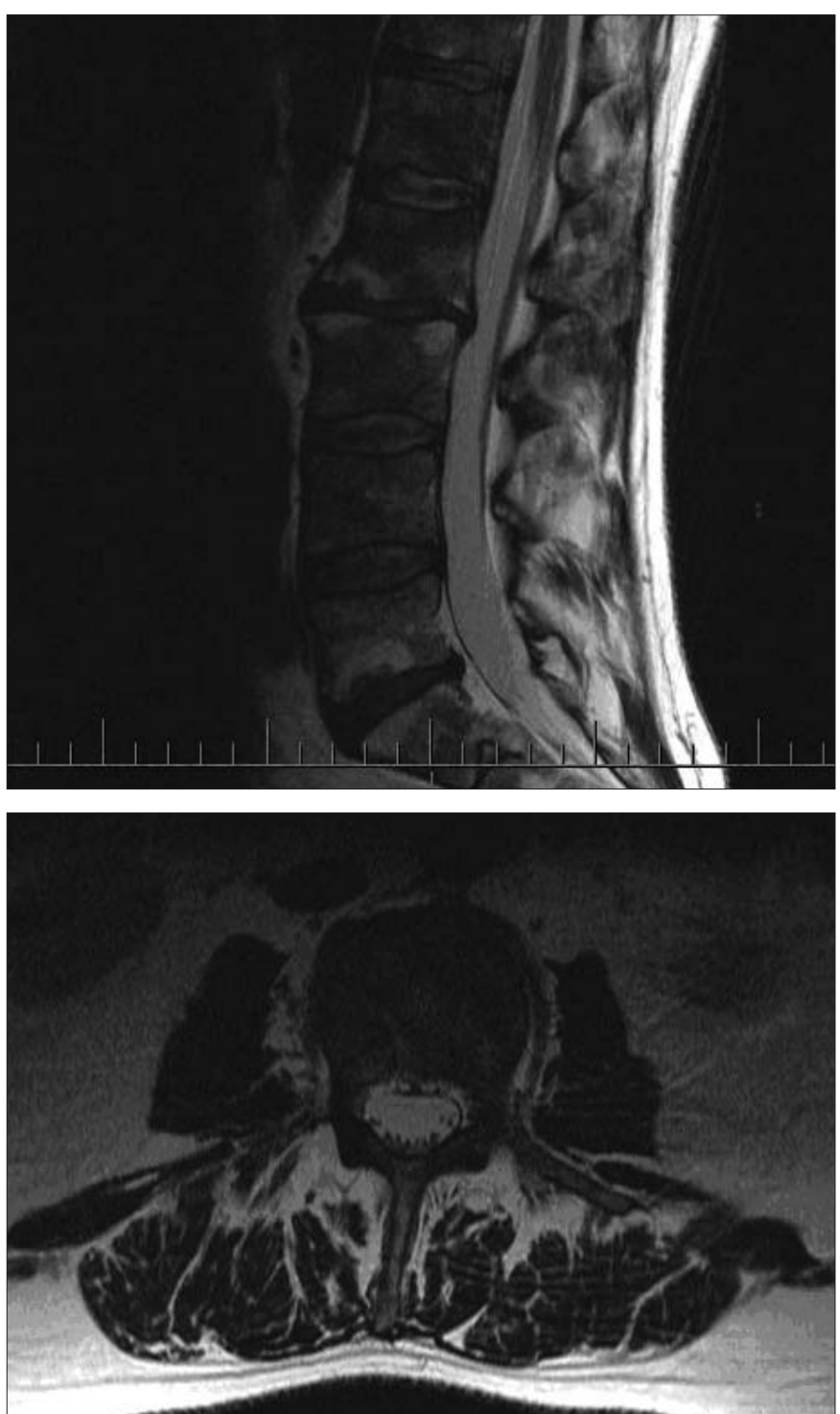

Fig. 3. Sagittal $T_{2}$-weighted magnetic resonance image (MRI) of the lumbar spine demonstrating normal anatomy of the conus medullaris and cauda equina (top). Axial $T_{2}$-weighted MRI of the lumbar spine demonstrating normal anatomy of the conus medullaris and cauda equina (bottom) (August 2006). 
The abnormal appearance of our patient's cauda equina on MRI may have been secondary to increased intrathecal pressure due to disrupted CSF flow caused by extensive epidural and vertebral disease. Several reports of intradural spinal neoplasms leading to increased intracranial pressure have been published, ${ }^{8-12}$ with 2 theories postulated to explain this. One involves elevated pressure due to increased CSF protein, which interferes with CSF absorption at the level of the arachnoid villi..$^{8,913}$ In our patient, this was unlikely as his lesions were epidural. The second attempts to explain increased intracranial pressure (ICP) in patients without elevated CSF protein, whereby tumours disrupt thecal sac elasticity and decompressive ability. ${ }^{14-16}$ This mechanical hypothesis is supported by a previous study that proposed a critical thecal sac size..$^{17,18}$ If the cross-sectional area of the thecal sac was reduced below this critical size, increased ICP occurred. This phenomenon may have occurred in our patient, given the anatomical disruption of his thecal sac.

Increased intrathecal pressure could also compromise venous outflow and arterial perfusion of peripheral nerves in a manner similar to the pathophysiology of SCC. ${ }^{10,19}$ This seems plausible in our patient's case as the cauda equina, compared with other peripheral nerve roots, has a poorly developed epineurium and lacks a segmental blood supply, making it highly susceptible to injury. ${ }^{17,20,21}$ The appearance of the cauda equina in our patient was unlikely to be a result of his 2004 pelvic radiation owing to the location of the nerve roots involved.

We cannot objectively confirm an increased opening pressure in our patient as we did not perform a lumbar puncture. However, he did not report classic symptoms of increased ICP such as headache, nausea or vomiting. It is possible, though, that the multilevel thoracic SCC could have prevented the propagation of increased intrathecal pressure cranially.

It is probable that the indirect cauda equina compression contributed to his clinical picture, as his symptoms could not solely be explained by his SCC. "Indirect" neurological symptoms have been reported in cancer patients previously. A 1999 case report described a patient with neoplastic meningitis with increased ICP and radicular pain relieved by CSF drainage..$^{10} \mathrm{~A}$ retrospective study of 362 patients with suspected metastatic SCC identified 5 patients with neurological symptoms and thecal compression but no structural evidence of metastases. ${ }^{19}$ No follow-up data for these patients is available.

One of the most striking features of our patient's case is the sudden development of profound asymmetrical LE weakness. Although it is possible that the thoracic compressions, along with tumour invasion into the right L4/L5 neural formina exacerbating the right leg weakness, contributed to his condition, the cauda equina compression probably played a role. A multifactorial etiology could best explain the asymmetry in the distribution and degree of weakness, as lower motor neuron lesions tend to cause more severe and flaccid weakness compared with upper motor neuron lesions. ${ }^{17}$ Additionally, the L2-L4 motor nerve (upper cauda equina) may have been more severely compromised than the middle and lower nerve roots, explaining his increased weakness proximally compared with distally. ${ }^{22}$

Another interesting aspect of our patient's case is the distribution of his sensory deficits. A sensory level starting well above the nipple line (T1/T2 level) or near the umbilicus (T8-T10 level) would correlate with MRI findings.
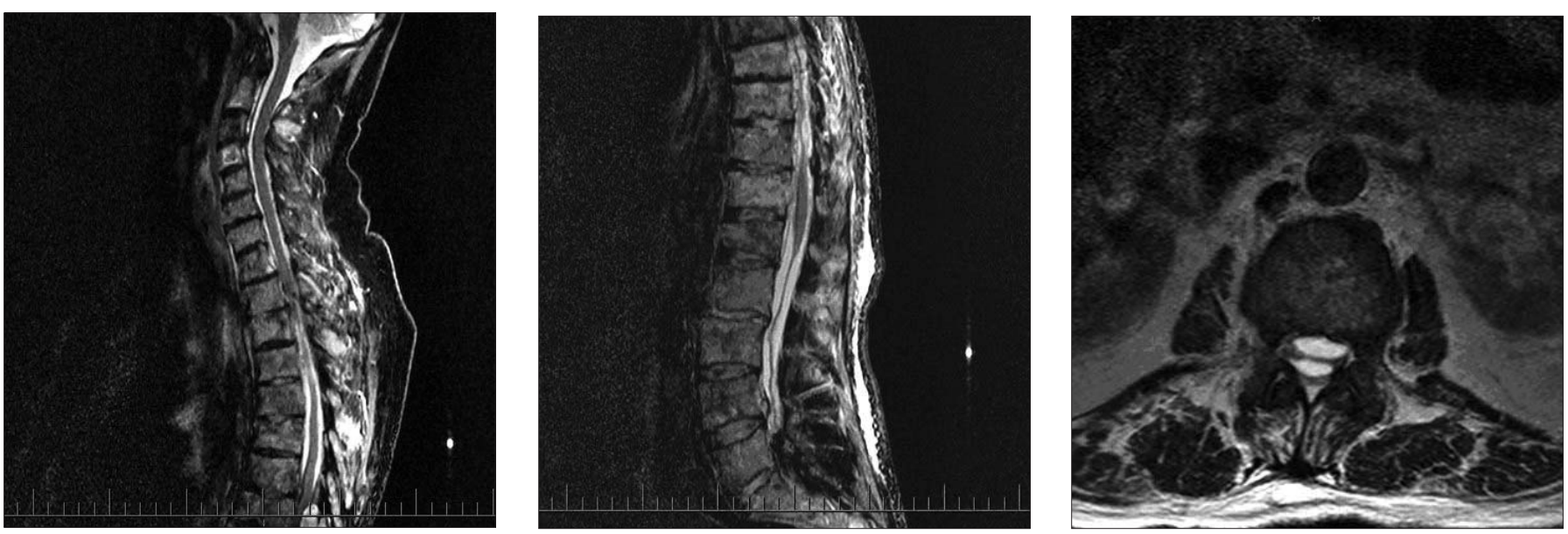

Fig. 4. Sagittal Short T, Inversion Recovery (STIR) sequence magnetic resonance image (MRI) of the thoracic spine demonstrating spinal cord compression at the $\mathrm{T} 1, \mathrm{~T} 2, \mathrm{~T} 8, \mathrm{~T} 9$ and T10 vertebral levels with impending compression at the T11 and T12 vertebral levels (left). Sagittal STIR sequence MRI of the lumbar spine demonstrating distortion of the cauda equina without direct tumour compression. On axial imaging, it appeared the cerebrospinal fluid (CSF) was compressing the cauda equina in the anteroposterior plane (middle). Axial $T_{2}$-weighted MRI demonstrating abnormal clumping of the cauda equina centrally (right) (March 2007). 
However, sensation was impaired bilaterally below the knees at the time of his second presentation. It is wellknown that sensory levels do not always correlate well with the level of compression. ${ }^{19,23-25}$ In SCC, sensory abnormalities begin distally and ascend to form a distinct sensory level. ${ }^{7}$ Additionally, lesions below the conus medullaris have been reported to cause symptoms earlier than lesions above it. ${ }^{26}$ Despite our patient's lack of saddle anesthesia (commonly seen in CES), these clinical observations suggest that impingement of the L5-S2 nerves was probably more directly responsible for the manifestation of his LE sensory changes than the thoracic SCC.

The cause of the hyperreflexia and urinary dysfunction were also probably multifactorial. The former was likely a result of an upper motor neuron lesion as the patient experienced hyperreflexia with his first SCC that did not resolve. It is difficult to determine the degree to which the new thoracic lesions contributed to our patient's condition. Compression of either the thoracic spinal cord or sacral nerves could be responsible for his urinary symptoms. ${ }^{27}$ One might expect that if the bladder dysfunction was caused by involved S2-S4 nerve roots, our patient would have had saddle anesthesia. His overflow incontinence secondary to retention is more consistent, however, with a neurogenic bladder from sacral nerve injury than the urge incontinence that typically develops from a spastic bladder due to a spinal cord lesion. ${ }^{28}$

The patient's functional recovery after his second course of RT was minimal and, although this is not surprising given the poor prognosis of SCC, ${ }^{5,7}$ it also implies a contribution from the untreated CES. This raises the question of whether treatment should be considered in these rare circumstances and, if so, with what approach and modality.

\section{Conclusion}

Malignant SCC remains a devastating manifestation of progressive metastatic disease in cancer patients. Our patient's case highlights the challenge of predicting the anatomical level of compression based on clinical presentation and reinforces the importance of imaging the entire spine with MRI. Our patient's previous treatment for SCC, multilevel involvement and atypical radiological appearance make it difficult to determine the cause of each of his signs and symptoms, with the amalgamation of all of the lesions, including the indirect cauda equina compression, producing the unusual clinical picture.

From the Departments of *Radiation Oncology and Medical Oncology, Cross Cancer Institute, Edmonton, Alta.

Acknowledgements: Ms. Lefresne was supported by the Alberta Cancer Research Institute and by an unrestricted educational grant from Ortho-Biotech.
This article has been peer reviewed.

Competing interests: None declared.

\section{References}

1. Coleman RE. Metastatic bone disease: clinical features, pathophysiology and treatment strategies. Cancer Treat Rev 2001;27:165-76.

2. Saad F, Clarke N., Colombel M. Natural history and treatment of bone complications in prostate cancer. Eur Urol 2006;49:429-40.

3. Bagley CA, Gokaslan ZL. Cauda equina syndrome caused by primary and metastatic neoplasms. Neurosurg Focus 2004:16:e3.

4. Ampil FL, Caldito G, Heldmann M. Palliation and survival in metastatic tumours compressing the cauda equina: A 19-year radiotherapeutic experience. J Palliat Med 2003;6:33-6.

5. Abrahm JL. Assessment and treatment of patients with malignant spinal cord compression. I Support Oncol 2004;2:377-88.

6. Bickley LS, Szilaqyi PG. Bates' Guide to Physical Examination and History Taking. 9th ed. Philadephia: Lippincott Williams \& Wilkins; 2007.

7. Cole JS, Patchell R. Metastatic epidural spinal cord compression. Lancet Neurol 2008;7:459-66.

8. Costello $F$, Kardon R, Wall M, et al. Papilledema as the presenting manifestation of spinal schwannoma. J Neuroophthalmol 2002;22:199-203.

9. Ghazi NG, Jane J, Lopes $M$, et al. Capillary hemangioma of the cauda equina presenting with radiculopathy and papilledema. J Neuroophthalmol 2006;26:98-102.

10. Groves MD, McCutcheon I, Ginsberg L, et al. Radicular pain can be a symptom of elevated intracranial pressure. Neurology 1999;52:1093-5.

11. Kounin GK, Romansky K, Traykov L, et al. Primary spinal melanoma with bilateral papilledema. Clin Neurol Neurosurg 2005;107:525-7.

12. Ridsdale L, Moseley I. Thoracolumbar intraspinal tumours presenting with features of raised intracranial pressure. J Neurol Neurosurg Psychiatry 1978;41:737-45.

13. Rekate HL. Why would a spinal tumour cause increased intracranial pressure? J Neuroophthalmol 2002;22:197-8.

14. Matzkin DC, Slamovits T, Genis I, et al. Disc swelling: a tall tail? Surv Ophthalmol 1992;37:130-6.

15. Ammerman BJ, Smith D. Papilledema and spinal cord tumors. Surg Neurol 1975;3:55-7.

16. Breen LA. Disc edema and peripheral neuropathy. Surv Ophthalmol 1994;38:467-74.

17. Orendacova J, Cizkova D, Kafka J, et al. Cauda equine syndrome. Prog Neurobiol 2001;64:613-37.

18. Schonstrom N, Bolender N, Spengler D., et al. Pressure changes within the cauda equina following constriction of the dural sac. Spine 1984;9:604-7.

19. Husband DJ, Grant K, Romaniuk C. MRI in the diagnosis and treatment of suspected malignant spinal cord compression. Br J Radiol 2001;74:15-23.

20. Lundborg G. Structure and function of the intraneural microvessels as related to trauma, edema and nerve function. J Bone Joint Surg Am. 1975;57:938-48.

21. Parke W, Gammell K, Rothman R. Arterial vascularization of the cauda equina. J Bone Joint Surg Am. 1981;63A:53-62.

22. Jaradeh S. Cauda equina syndrome: A neurologist's perspective. Reg Anesth 1993;18(Suppl):473-80.

23. Cook AM, Lau TN, Tomlinson MJ, et al. Magnetic resonance imaging of the whole spine in suspected malignant spinal cord compression: Impact on management. Clin Oncol (R Coll Radiol)1998;10:39-43.

24. Levack P, Graham J, Collie D, et al. Don'† wait for a sensor level - listen to the symptoms: A prospective audit of the delays in diagnosis of malignant spinal cord compression. Clin Oncol (R Coll Radiol) 2002; 14:472-80.

25. Loughrey GJ, Collins C, Todd S, et al. Magnetic resonance imaging in the management of suspected spinal canal disease in patients with known malignancy. Clin Radiol 2000;55:849-55.

26. Boogerd W, Van der Sande J, Kroger R. Early diagnosis and treatment of spinal epidural metastasis in breast cancer: a prospective study. J Neurol Neurosurg Psychiatry 1992;55:1188-93.

27. Fowler CJ. Neurological disorders of micturition and their treatment. Brain 1999;122:1213-31.

28. Williams 0 , Stern M. Nontraumatic disorders of the spinal cord. In: Brust J. Current Diagnosis and Treatment in Neurology. New York: McGraw Hill Companies Inc, 2007, pp 262-280.

Correspondence: Dr. Alysa Fairchild, Department of Radiation Oncology, Cross Cancer Institute, 11560 University Ave., Edmonton AB T6G 122; fax 780 432-8380; alysafai@cancerboard.ab.ca 